\title{
Information flow and gaps
}

\author{
P.L.A. Piwek \\ e-mail: paul.piwek@itri.brighton.ac.uk
}

\begin{abstract}
In this paper we provide an outline of a model for the flow information in conversations. We explain the model by means of a metaphor. We propose that certain natural language phrases (i.e., anaphoric expressions and questions) are used to express gaps. These gaps are what gives rise to information flow in conversations. After dealing with a simple conversational exchange in terms of this approach, we proceed to deal with different forms of indirect information transfer in conversations as they are manifested by bridging anaphors and indirect answers. At that point, we will leave the metaphorical presentation behind us and examine some of the details of our logic-based model itself. For the full details of the model we refer to Piwek (1998).
\end{abstract}

\section{Introduction}

The success of an agent depends heavily on the ability of the agent to adapt its behaviour to its environment. Adaptation can be achieved by means of direct stimulus-response relations between events in the environment and actions of the agent. For instance, snails withdraw into their shell when they come into contact with a pointed object. Such behaviour is very much tied to the local properties of the current situation in which the agent finds itself. Often it is, however, profitable to take into account information which goes beyond the confines of the current situation. For instance, for deciding whether to go to the cinema right now, information I came across when reading the programme some time ago is certainly helpful. This presupposes that I stored the information that is conveyed by the programme in some form or other. This information can be considered to be an internal representation, which can guide an agent in its interaction with the environment that the representation stands for.

The internal representation that an agent has of the world is constantly updated in various ways (which might actually not be as disparate as the following classification suggests). We may discern direct observation, theory building (i.e., generalizing information which came from direct observations), drawing conclusions and communication, i.e., exchange of information amongst agents. When agents communicate, they aim at sharing the representations that they individually have of their environment (this environment includes the agents themselves and their conversation) ${ }^{1}$. Of course, sharing of information is only feasible if the agents entertain structurally similar conceptualizations of the world.

The aim of this paper is to provide the outline of a formal model of information flow in natural language dialogue (the paper is based on Piwek (1998) which contains more details. See also the last section of this paper for related references). This model should predict whether a particular dialogue is felicitous, given the background in which the dia-

1. Even utterances such as 'hello' can be seen as conveying information. For instance, 'hello' can be used to indicate that an agent wants to initiate a conversation.

IPO Annual Progress Report 331998 
logue is conducted. On the basis of our functional view on the role of information, we can formulate an a priori constraint on our model of information flow: information flow should not destroy the representational nature of the information. If this representational nature is lost, the information can no longer help the agent to adapt to its environment.

At this point, logic, i.e., the study of correct reasoning, comes into the picture. Logic concerns precisely the manipulation of information without loss of its representational nature: an argument is logically valid only if the truth of premises carries over to the truth of the conclusion. We shall see that logic is not only on a priori grounds a good candidate for modelling information flow, but also provides an adequate model for the data of natural language use.

We will proceed as follows. We start by providing a metaphorical account of information flow. This account is developed by discussing a very simple example. Subsequently, we discuss some of the details of the underlying formal model. This enables us to provide an outline of our account of 'indirect' information flow in discourse. The main aim will be to show that logic provides the proper handle to deal with that type of information flow. Finally, we end with a summary and some references to further reading on the topic of this paper.

\section{Gaps and changing pools of information}

To speak of information flow would be somewhat gratuitous if there were not some outstanding characteristics that information shares with other things that can flow, such as, say, water, tea, or coffee. The model of information that we propose includes such a characteristic. Like a liquid, information can be thought of as flowing from higher to lower places; in particular, it flows into informational gaps. This analogy between information and liquids should become clearer after we have introduced the notion of an informational item.

\section{Informational items}

An informational item consists of an object, and this object carries its type with it. Thus, instead of writing $\mathrm{o}$, for some informational object, we write $o: t$, where $t$ is the type of the object $o$. The type should be thought of as part of a conceptualization of the external world, i.e., the domain of interpretation of the informational items. The presence of an object of a certain type $t$ in the agent's information pool means to the agent that there exists an object in the external world which fits the type $t$. To make this more concrete, let us have a look at a very simple body of information. The domain of interpretation for this pool of information is an electron microscope ${ }^{2}$. This pool of information contains five items (to the right, we have added natural language paraphrases of each of the informational items):

(1)

$\begin{array}{ll}a: \text { lens } & \text { there is a lens, we refer to it with constant } a \\ b: \text { lens } & \text { there is a lens, we refer to it with constant } b \\ s: c 1 \cdot a & a \text { is a c1 lens } \\ t: c 2 \cdot b & b \text { is a c2 lens } \\ u: \text { excited } \cdot b & b \text { is excited }\end{array}$

2. The example is taken from the DENK domain (see Bunt et al., 1998). 
In words: There are two lenses, $a$ and $b$. They are different kinds of lenses: $a$ is a c1 lens, and $b$ is a c 2 lens. Furthermore, $b$ is excited (The light source in an electron microscope is an electron gun. The microscope has electromagnetic lenses. By varying the current through the lens, the magnification can be changed). Note that in our example, there are also objects whose type is a proposition (e.g., $u$ in $u$ : excited $\cdot b$ ). Such an object stands for the situation which would make the proposition true in the domain of interpretation. Simultaneously, the object functions as an internal proof/witness for the agent that the proposition holds.

\section{Information flow in dialogue}

A dialogue enables the interlocutors to share the information that their respective pools of information contain. The information pool of an agent can be divided into the information that the agent believes to share with the other agent, let us call this its public information, and the information that the agent does not believe to share, i.e., its private information. In the course of a dialogue, private information is continually turned into public information. The prototypical conversational unit for doing so is a pair of utterances, such as a question followed by an answer. By means of a question, an interlocutor can specify what information it would like to become public. The answer subsequently provides this information $^{3}$. Consider:

$$
\begin{aligned}
& \text { A : Which lens is excited? } \\
& \text { B : The c2 lens (is excited). }
\end{aligned}
$$

We will examine this exchange in detail by assigning a pool of information to each of the interlocutors at the outset of the exchange, and then study how their utterances cause that information to start flowing. At the beginning of the dialogue, interlocutor B has the information pool under (1). Additionally, assume that in B's pool of information, the information that there is a $\mathrm{c} 1$ and a $\mathrm{c} 2$ lens is considered to be public, whereas the information that the $\mathrm{c} 2$ lens is excited is private.

So, let us examine A's utterance from B's perspective. A's utterance can be seen as presenting an informational gap to B. This gap has to be filled with a lens which is excited. Formally, such gaps can be expressed by means of a so-called segment:

$$
\boldsymbol{X}: \text { lens, } S: \text { excited } \cdot \boldsymbol{X}
$$

The bold faced $\boldsymbol{X}$ stands for the gap that needs to be filled. Note that there is another gap, $S$, which also needs to be filled. The latter gap has to be filled with a proof for the proposition that the filler of $\boldsymbol{X}$ is excited. However, there is a crucial difference between $\boldsymbol{X}$ and $S$ : whereas the person who posed the question is interested in the identity of the filler of $\boldsymbol{X}$, the identity of $S$ is irrelevant ${ }^{4}$. The pool of information which is associated with B does indeed provide the information to fill these gaps:

3. For the purpose of this paper, we make the simplifying assumption that the person who answers the question is known, by the interlocutors, to be an expert on the topic of the question. This causes the answer to become shared information. For a more sophisticated model of how information comes to be shared, see, e.g., Piwek (1998). 
(4)

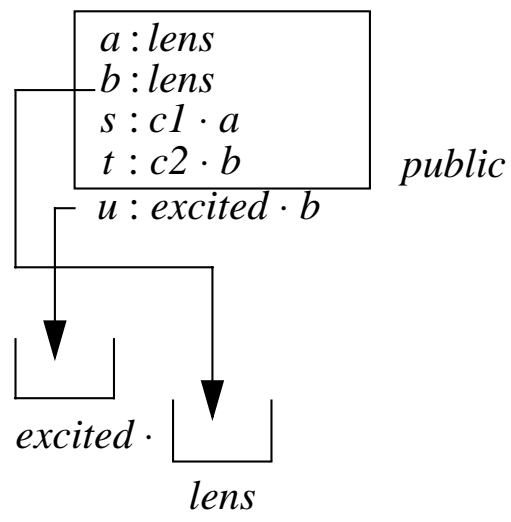

Here, we have used a pictorial representation for the gaps $\boldsymbol{X}$ and $S$. Each gap has, what we might call, a filter associated with it. The filter is written below the gap and determines which objects are allowed into the gap. In this case, the gaps $S$ and $X$ are filled with the objects $u$ and $b$, respectively. We already pointed out that from the questioner's point of view only the filler of gap $\boldsymbol{X}$ is of interest: this filler stands for the lens which is excited. At this point, the task for $\mathrm{B}$ is to communicate to A that the object which $b$ represents is excited.

If B has a correct representation of the public information, then A's representation of the public information will also contain an object for the $\mathrm{c} 1$ lens and an object for the c2 lens:

$$
\begin{array}{ll}
c & : \text { lens } \\
d & : \text { lens } \\
v & : c 1 \cdot c \\
w & : c 2 \cdot d
\end{array}
$$

B's response ('the c2 lens is excited') can be seen as being composed of two parts: the identification of an object ('the c2 lens') and the assertion that this object is excited. The identification of the object is achieved by means of a gap; 'the c2 lens' corresponds to:

$$
Y: \text { lens, } T: c 2 \cdot Y
$$

The fillers for the gaps $Y$ and $T$ are $d$ and $w$, respectively. Secondly, B's utterance contains new information about the object $d$. Assuming that in this conversation $\mathrm{B}$ is an expert on lenses, A and B will make the proposition that this lens is excited public information. B can achieve this by simply moving ' $u$ : excited $\cdot b$ ' from its private to its public information. In the case that A was asking a genuine question (i.e., not an exam question or a rhetorical question), A's private information did not yet contain a proof for the fact that $d$ is excited. Therefore, A has to add a fresh proof for that proposition to its representation of the public information. In summary, after the exchange in (2), the public information has been updated with the information that the $\mathrm{c} 2$ lens is excited.

4. $S$ needs to be filled with an object which stands for a situation, i.e., the situation in virtue of which excited $\cdot x$ (for some object $x$ ) is true. If excited $\cdot x$ is true, there is exactly one such situation. Thus, the identity of that situation cannot be something which the questioner is interested in. 


\section{Logic and indirect information}

In order to deal with more complicated cases of information flow, we need to introduce some formal notation. Let $\Gamma_{\mathrm{A}}$ and $\Gamma_{\mathrm{B}}$ stand for the pools of information of A and B, respectively. Now, reconsider (4). There we provided a pictorial representation of the filling of two gaps, i.e., $\boldsymbol{X}$ and $S$ in the segment $\boldsymbol{X}$ : lens, $S$ : excited $\cdot \boldsymbol{X}$. Henceforth, we will write the following to state that the gaps in a segment $\Delta$ can be filled given some pool of information $\Gamma$ :

$$
\text { There is a substitution }[\sigma] \text { such that: } \Gamma \vdash \Delta[\sigma]
$$

A substitution $[\sigma]$ is an assignment of objects to the gaps in a segment. For instance, the substitution that is represented in (4) is written $[X:=b, S:=u]$. In its entirety (4) corresponds to:

$$
\Gamma_{\mathrm{B}} \vdash \boldsymbol{X}: \text { lens, } S: \text { excited } \cdot \boldsymbol{X}[X:=b, S:=u] .
$$

If we carry out the substitutions in (8) we obtain:

$$
\Gamma_{\mathrm{B}}+b: \text { lens, } u: \text { excited } \cdot b
$$

Logic enters the picture through the relation ' $~$ '. Alternative ways to read ' $\Gamma+C$ ' are: ' $C$ can be derived in $\Gamma$ ' and ' $C$ is deducible from $\Gamma$ '. In the examples that we presented up till now, something could be deduced from a pool of information if it was literally present in that pool of information. Logic, however, also allows us to make information that is implicitly present explicit. Consider, for instance, the following two pieces of information:

(10) (a) If Mary's car is in the garage, then she is at home.

(b) Mary's car is in the garage.

A pool of information which contains these two pieces of information, implicitly contains the information that Mary is at home. If we abbreviate the propositions expressed by (10.a) and (10.b) as ' $p \Rightarrow q$ ' and ' $p$ ', respectively, then the formal representations of the corresponding informational items are:

$$
\begin{aligned}
& \text { (a) } f:(p \Rightarrow q) \\
& \text { (b) } a: p
\end{aligned}
$$

In other words, there is a proof $f$ for the proposition $(p \Rightarrow q)$ and a proof $a$ for the proposition $p$. A proof for $(p \Rightarrow q)$ corresponds to a function which yields a proof for $q$ when it is applied to a proof for $p$. Thus, $f$ can be used to obtain a proof for $q$ by applying $f$ to $a$. This way, we obtain the proof $f \cdot a$ for $q$ (where '.' stands for function application). In other words, a proof for 'Mary is at home' can be constructed from the information that is conveyed by (10.a) and (10.b).

\section{Presupposition}

When we dealt with the utterance of 'The c2 lens is excited', we pointed out that 'the c2 lens' gives rise to a gap which has to be filled with an object from the public information. 
We pointed out that the identification of this object is separate from the assertion that is performed by uttering 'The c 2 lens is excited'. This observation goes back to Frege (1892), one of the founding fathers of modern logic, who pointed out that negation does not affect the information that is conveyed by a definite description such as 'the $\mathrm{c} 2$ lens':

The $\mathrm{c} 2$ lens is not excited.

Whereas the negation does reverse the assertion, (12) still requires that the public information contains a c2 lens. Expressions whose interpretations behave this way are called presuppositions. Let us now look at some properties of presuppositions, which nicely fit into the model of information flow that we have proposed.

We start by an examination of presuppositions in conditionals. Compare the discourse in (13.a) and (13.b):

(13.a) John has a bottle of Châteauneuf-du-Pape. He keeps it in his wine cellar. The bottle is twelve years old.

(13.b) (??) If John has a bottle of Châteauneuf-du-Pape, then he keeps it in his wine cellar. The bottle is twelve years old.

The two question marks indicate that the discourse in (13.b) is intuitively felt to be anomalous as opposed to the felicitous discourse in (13.a), which differs only slightly from (13.b). The difference between (13.a) and (13.b) is that in (13.b) the object which is presupposed by the utterance of 'the bottle' is introduced in the antecedent of a conditional (i.e., 'If John has a bottle of Châteauneuf-du-Pape'). It seems that this object is not accessible for reference in the sentence which follows the conditional. Note, however, that the bottle is accessible from the consequent of the antecedent, as is witnessed by the fact that the description 'the bottle' in (14) picks the object which is introduced as 'a bottle of Châteauneuf-du-Pape':

(14) If John has a bottle of Châteauneuf-du-Pape, then he keeps the bottle in his wine cellar.

We model the judgments concerning (13) and (14) by positing that an object which is introduced in the antecedent of a conditional is not added to the public information, but rather treated as a hypothetical extension of the public information. This hypothetical extension is only used for the interpretation of the consequent of the conditional. Therefore, the object is no longer accessible in the discourse that succeeds the conditional.

The felicity of (13.a) requires no further explanation. It follows from our approach to presuppositions as it has been sketched in the previous section. So, let us turn to (14). The structure of (14) can be represented as:

$$
P \Rightarrow Q_{\pi}
$$

Here, $\pi$ stands for the gap that is induced by the description 'the bottle' (formally we write: $[X:$ object, $P:$ bottle $\cdot X])$. According to our approach, $\pi$ has to be filled with respect to $\Gamma$ (the public information of the information pool of the interpreter) extended with $P$, 
i.e., a substitution $S$ is required such that:

$$
\Gamma, P \vdash \pi[S]
$$

There is indeed such a substitution. This substitution assigns the object that is introduced by 'a bottle of Châteauneuf-du-Pape' in $P$, to the gaps in $\pi$. Now compare this with the interpretation of the last sentence in (13.b). It has to be interpreted with respect to $\Gamma$ only. $\Gamma$ does not contain the introduction of a bottle, which is presupposed by the utterance of 'the bottle' in the last sentence. Hence, the infelicity of the discourse.

Let us now consider another small discourse, which provides further evidence for the role of logical inference in information flow (the example is taken from Kamp and Reyle, 1993, p.205):

(a) The barn contains a chain saw or a power drill.

(b) It makes an ungodly racket.

Intuitively, 'It' in (17.b) refers to a machine, whether it be a chain saw or a power drill, which is present in the barn that has been introduced in (17.a). Now, the problem is that such a machine has not been mentioned explicitly in the first sentence of (17.a). We will show that the information about the machine is, however, implicit in the information that is conveyed by (17.a) and can be made explicit by means of logical inference and the use of some world knowledge.

The piece of world knowledge that is required to interpret 'It' in (17.b) is that chain saws and power drills are machines. This piece of world knowledge is public information and can, therefore, be used by an interpreter of (17). Thus, we assume that the public information of a pool of information contains more than solely the information which has been exchanged in the current discourse. It also contains all information which can reasonably be assumed to be public on the basis of the fact that the interlocutors are members of the same community of language users.

In order to demonstrate the logical inference from the explicit information conveyed by (17.a) to the implicit information, we need to introduce some abbreviations:

$$
\begin{aligned}
& p=\text { There is a chain saw in the barn. } \\
& q=\text { There is a power drill in the barn. } \\
& r=\text { There is a machine in the barn. }
\end{aligned}
$$

Now let us first restate the world knowledge that is needed:

$$
\begin{aligned}
& \text { (a) } p \Rightarrow r \\
& \text { (b) } q \Rightarrow r
\end{aligned}
$$

The content of (17) itself is $p$ or $q$. Our aim is to prove that $r$ holds. We proceed as follows. We assume that $r$ does not hold (i.e., not $r$ ) and proceed to prove that this assumption leads to a contradiction. From that, we conclude that $r$ does hold (this patterns of logical argumentation is called the reductio ad absurdum). So, assume that not $r$. From (19.a) and not $r$, it follows that not $p$ (this inference step is called contraposition). Similarly, from (19.b) and not $r$, it follows that not $q$. From (17) and not $q$, we can prove that $p$. Note that we had 
already proven not $p$. Hence, the assumption not $r$, on the basis of which we proved this contradiction, is false. In other words, $r$ is true ${ }^{5}$.

\section{Questions and answers}

We showed that questions can also be seen as introducing a gap which needs to be filled by an object from the public information. Again, the role of inference and background knowledge is paramount. Consider:

$$
\begin{aligned}
& \text { A : Who is at home? } \\
& \text { B : Mary's car is in the garage. }
\end{aligned}
$$

At face value, B's utterance does not provide a filler for the gap that corresponds to A's question. The gap has to be filled with a person who is at home. But now, assume that the pool of information against which A evaluates B's answer contains the information that 'If Mary's car is in the garage, then she is at home'. In that case, the filler can be deduced; for details, see the discussion of (10). Alternatively, consider:

$$
\begin{aligned}
& \text { A : Who is at home? } \\
& \text { B : If Mary's car is in the garage, then she is at home. }
\end{aligned}
$$

Again, no filler is presented directly. In this case, a filler (i.e., 'Mary') could be deduced if the information that Mary's car is in the garage is already available. But also if this information is not available, B's response seems to be a genuine answer to A's question. Here the notion of a hypothetical extension of the information pool, which we already used for modelling presuppositions, is useful again. Whereas a direct answer leads to a filler of the question by simply adding it to the informational pool, an indirect answer, as in (21), provides a filler only with respect to an additional hypothetical extension of the information pool (for (21) this is the information that Mary's car is in the garage). The hypothetical extension can be read from the structure of the answer: if the answer is a conditional, the extension is the antecedent of the conditional. Such answers will be called conditional answers. Unfortunately, this structural approach is to crude. Consider:

$$
\begin{aligned}
& \mathrm{A}: \text { Is the TV on? } \\
& \mathrm{B}: \text { It is plugged in. }
\end{aligned}
$$

Now, assume that the background information which is available is: If the TV is plugged in and the on switch has been pressed, then the TV is on. Note that in this case, B's answer is neither a direct nor a conditional answer. Fortunately, we can use the notion of conditional answerhood as the basis of a notion of indirect answerhood which covers (22). We define an indirect answer as an answer which, when it is added to the information pool, allows us to derive a new conditional answer. In this case, the new conditional answer is: If the on switch has been pressed, then the TV is on.

5. Note that at the end of this chain of reasoning we employed the principle of the excluded middle, i.e., $p$ is either false or true. That allowed us to infer $r$ from the falsity of not $r$. In intuitionistic mathematics (as opposed to classical mathematics), this principle is not valid. In our view, examples such as (18) strongly suggest that the principle is relied on in ordinary language use. 
We do, however, have to be careful. As it stands, this definition turns every response into an indirect answer (Groenendijk and Stokhof (1984) point out a similar problem for their modeltheoretic account of indirect answerhood). Consider:

$$
\begin{aligned}
& \text { A : Is John at home? } \\
& \text { B : My cup is empty. }
\end{aligned}
$$

The conditional answer that can be inferred after adding B's response to the pool of information is: 'John is at home if the fact that my cup is empty implies that John is at home'. The antecedent of this conditional answer (i.e., the fact that my cup is empty implies that John is at home) is, however, rather peculiar. The antecedent and the actual answer already suffice to find a filler for A's question. In other words, the antecedent is overinformative; the information that is required to prove the antecedent is equivalent to the information conveyed by the consequent.

Note the difference with the answer in (21). There the antecedent of the conditional answer contains information which is different from the information in the consequent. This means that the questioner can find an answer to his original question ('Who is at home'), by inspecting whether Mary's car is in the garage. He does not have to find out whether Mary is at home, to have his question answered. Therefore, the answer in (21) is genuinely helpful, whereas the one in (23) is not. For a formal account of indirect answerhood we refer to Piwek (1998).

\section{Summary and further reading}

The main aim of this paper is to demonstrate the usefulness of logic for the study of conversation. In this respect, our aims are very much in line with Grice's work on logic and conversation (Grice, 1975; 1989). Grice tried to show that the apparent divergences between certain formal devices, such as $\wedge, V, \Rightarrow$ and their counterparts in natural language (and, or, if ... then ...) are illusive, and disappear when we carefully study the conditions governing conversations.

Of course, things have changed since Grice initiated his programme. In particular, whereas Grice studied the relation between logic and conversation, nowadays it has become a truism that the two only come together through the study of information and, in particular, information flow. Information flow is central to this paper. We show that information can indeed be said to flow in some sense: it flows into gaps. We address some problems in the treatment of presuppositions and questions, and show that the use of gaps and a good deal of logic gets us quite some way.

The notion of information that is used in this paper goes back to the pioneering work of Karttunen (1976). He was probably the first to see indefinites as introducing objects which under certain conditions are available for later reference. The ideas that were already present in Karttunen's work were first formalized by Heim (1982) and Kamp (1981). The work has become known as 'File Change Semantics' and 'Discourse Representation Theory' (DRT), respectively. Interestingly, the formal frameworks set up by Kamp and Heim are similar to a class of formal systems, Constructive Type Theories (e.g., Martin-Löf, 1984; De Bruijn, 1984; Nederpelt, Geuvers \& De Vrijer, 1994), which predate them. Sundholm (1986) was the first to use Constructive Type Theory (CTT) to model some of the data that are also covered by DRT. 
Other researchers who have employed CTT for modelling natural language are, notably, Ranta (1994) and Krause (1994). The core of this paper is based on Piwek (1998), who also uses CTT as his formal framework. The research on which this paper was based was carried out within the DENK project (Bunt et al., 1998). Within that project, CTT was used as the formal basis of a dialogue system. A selection of further papers on information exchange and CTT which arose out of this project are Ahn (1995), Ahn and Borghuis (1997), Beun and Kievit (1995), Kievit (1998), Krahmer and Piwek (forthcoming), and Piwek and Krahmer (in press). Finally, we refer to Beun (1994) and Piwek (1997; 1998) for further investigations of dialogue structure and information flow within DENK.

\section{Acknowledgements}

Thanks are due to Robbert-Jan Beun and Adam Kilgarriff for helpful comments on an earlier version of this paper. The research reported in this paper was financially supported by the Cooperation Unit of Brabant Universities (SOBU).

\section{References}

Ahn, R.M.C. (1995). Communicating contexts: A pragmatic approach to information exchange. In: P. Dybjer, B. Nordström and J. Smith (Eds.): Types for Proofs and Programs. International Workshop TYPES-94, Båstad, Sweden, June 6-10, 1994, Selected Papers. Lecture Notes in Computer Science 996, 1-14. Berlin: Springer Verlag.

Ahn, R.M.C. \& Borghuis, V.A.J. (1997). Communication modelling and context-dependent interpretation: An integrated approach. In: A. Benz and G. Jäger (Eds.): Proceedings of the Munich Workshop on Formal Semantics and Pragmatics of Dialogue, MunDial-97, Munich, Germany, March 10-12, 1997, 2-20. CIS 97-106. Universität München: Centrum für Informations- und Sprachverarbeitung.

Beun, R.J. (1994). Rules in dialogue. In: M.D. Brouwer and T.L. Harrington (Eds.): Human-Machine Communication for Educational Systems Design. NATO ASI Series F, Volume 129, 79-88. Berlin: Springer-Verlag.

Beun, R.J. \& Kievit, L.A. (1995). Resolving definite expressions in DENK. DENK Report 95/16. Tilburg, Eindhoven: SOBU.

Bruijn, N.G. de (1984). A survey of the project Automath. In: J.R. Seldin and J.P. Hindley (Eds.): To H.B. Curry: Essays on Combinatory Logic, Lambda Calculus and Formalisms, 589-606. London: Academic Press.

Bunt, H.C., Ahn, R.M.C., Kievit, L.A., Piwek, P.L.A., Verlinden, M.E.M.C., Beun, R.J. \& Borghuis, V.A.J. (1998). Multimodal cooperation with the DENK system. In: H. Bunt, R.J. Beun, T. Borghuis, L. Kievit and M. Verlinden (Eds.): Proceedings of the 2nd International Conference on Cooperative Multimodal Communication, CMC-98, Tilburg, The Netherlands, January 28-30, 1998, 44-62.

Frege, G. (1892). Ueber Sinn und Bedeutung. Zeitschrift für Philosophishe Kritik, 100, 25-50. [Reprinted in: G. Patzig (Ed.) (1986). Funktion, Begriff, Bedeutung: Fünf logische Studien, 40-65. Göttingen: Vandenhoeck \& Ruprecht].

Grice, H.P. (1975). Logic and conversation. In: P. Cole and J.L. Morgan (Eds.): Syntax and Semantics, Volume 3: Speech Acts, 41-58. New York: Academic Press.

Grice, H.P. (1989). Studies in the Way of Words. Harvard: Harvard University Press.

Groenendijk, J.A.G. \& Stokhof, M. (1984). On the semantics of questions and the pragmatics of answers. In: F. Landman and F. Veltman (Eds.): Varieties of Formal Semantics, 143-170. Dordrecht: Foris.

Heim, I. (1982). On the Semantics of Definite and Indefinite Noun Phrases. PhD thesis, University of Amherst, MA, USA.

Kamp, J.A.W. (1981). A theory of truth and semantic interpretation. In: J. Groenendijk, T. Janssen and M. Stokhof (Eds.): Formal Methods in Study of Language, 277-322. Mathematical Centre Tracts 136. Amsterdam: Mathematisch Centrum. 
Kamp, J.A.W. \& Reyle, U. (1993). From Discourse to Logic. Dordrecht: Kluwer Academic Publishers. Karttunen, L. (1976). Discourse referents. In: J.D. McCawley (Ed.): Syntax and Semantics, Volume 7: Notes from the Linguistic Underground. New York: Academic Press.

Kievit, L.A. (1998). Context-Driven Natural Language Interpretation in Constructive Type Theory. PhD thesis, Tilburg University, Tilburg, The Netherlands.

Krahmer, E.J. \& Piwek, P.L.A. (forthcoming). Presupposition projection as proof construction. In: H. Bunt and R. Muskens (Eds.): Computing Meanings: Current Issues in Computational Semantics. Studies in Linguistics \& Philosophy Series. Dordrecht: Kluwer Academic Publishers.

Krause, P. (1995). Presupposition and abduction in type theory. In: E. Klein, S. Manandhar, W. Nutt and J. Siekmann (Eds.): Working Notes of the Conference on Computational Logic and Natural Language Processing, South Queensferry, Scotland, April 1995, no page numbers. Edinburgh: Human Communication Research Centre.

Martin-Löf, P. (1984). Intuitionistic Type Theory. Napels: Bibliopolis.

Nederpelt, R.P., Geuvers, J.H. \& Vrijer, R.C. de (Eds.) (1994). Selected Papers on Automath. Studies in Logic and the Foundations of Mathematics, Volume 133. Amsterdam: North-Holland.

Piwek, P.L.A. (1997). Situated action and commitment in dialogue. IPO Annual Progress Report, 32, 8997.

Piwek, P.L.A. (1998). Logic, Information \& Conversation. PhD thesis, Eindhoven University of Technology, Eindhoven, The Netherlands.

Piwek, P.L.A. \& Krahmer, E.J. (in press). Presuppositions in context: Constructing bridges. In: P. Brezillon and M. Cavalcanti (Eds.): Formal and Linguistic Aspects of Context. Dordrecht: Kluwer Academic Publishers.

Ranta, A. (1994). Anaphora in game theoretical semantics and in intuitionistic type theory. In: L. Haaparanta, M. Kusch and I. Niiniluoto (Eds.): Language, Knowledge and Intentionality: Perspectives on the Philosophy of Jaakko Hintikka, 265-274. Helsinki: Acta Philosophica Fennica.

Sundholm, G. (1986). Proof theory and meaning. In: D. Gabbay and F. Guenthner (Eds.): Handbook of Philosophical Logic, Volume 3, 471-506. Dordrecht: D. Reidel. 
In: IPO Annual Progress Report 33, Eindhoven University of Technology, 1998. 